\title{
Crew Size, Composition, and Time: Implications for Habitat and Workplace Design in Extreme Environments
}

\author{
Marilyn Dudley-Rowley \\ OPS-Alaska \\ Stewart Whitney \\ Niagara Univ. \\ Sheryl Bishop \\ University of Texas \\ Barrett Caldwell \\ Purdue Univ. \\ Patrick D. Nolan \\ University of South Carolina
}

Copyright () 2001 Society of Automotive Engineers, Inc.

\begin{abstract}
The authors report the results of an ongoing study that investigates the effects of crew size, composition, mission duration, and mission interval on behavior and performance among polar and space expeditions. The standardized rates for a behavior/performance indicator constructed during the pilot study displayed distinctive patterns across different crew profiles and settings. Then, a further analysis over the missions in the pilot sample found compelling information suggesting that several factors created specific differentials between outside (baseline) groups (e.g., mission controllers, "folks back home") and groups in extreme environments. These differentials reflected how the passage of time was subjectivized by crews and how the expeditionary situation was otherwise defined differently from baseline. These analyses suggest that the definition of the longduration mission, such as a mission to Mars, likely involves more than the issue of real-time duration. Also, crew size and composition of such ventures need to be examined in light of social and behavioral information that can be obtained from the expeditionary record. These analyses hold important implications for habitat and workplace design in extreme environments, such as those to be deployed for Mars surface operations.
\end{abstract}

\section{INTRODUCTION TO THE PILOT STUDY}

The psychosocial aspects of extreme environments fall in the area of human factors. However, rather than being primarily interested in the human-material culture or human-environment interfaces - which are the classical concerns of human factors science - the psychological and sociological aspects of extreme environments are interested in the human-human interface. Though a number of scientists have produced a compelling body of research at this interface, the realities of short-duration space missions and limited space budgets prevent the footing of any thorough and sustained effort in psychosocial investigation across all space-related organizations. These considerations account for most of the prior neglect in this area of study, and to some degree for the neglect in related biomedical concerns.

Tours of duty on the International Space Station and the prospect of long-duration space missions change everything. Various incidents have happened on recent space missions, on recent polar work teams, and in simulations. Actually, such incidents have been happening all along, as the expeditionary record bears out, but the implications of those incidents and sustained working and living on the high frontier have taken on a significance and urgency as never before. Suddenly, the realization dawns that we had better start paying attention to the psychosocial aspects of extreme environments and the related biomedical concerns.

This report presents the results of a pilot study investigating the effects of crew characteristics, mission duration, and mission interval on rates of deviance, 
conflict, and dysfunction among performance crews in extreme environments (i.e., space missions and polar expeditions). This study, which is preliminary to a more extensive project funded by the National Science Foundation, had three basic objectives. They were to:

1. Develop and test protocols for reliably coding deviance/conflict from written records (e.g., logs, diaries, and narratives) of space missions and polar expeditions;

2. Provide preliminary evaluation of a priori hypotheses concerning the impact of demographic features of crews, and of mission duration, on rates of deviance/conflict among performance teams in extreme environments; and

3. Provide preliminary evaluation of the anecdotal hypothesis that rates of deviance/conflict will peak just after mission midpoint (i.e., the "third-quarter phenomenon").

The first phase of the pilot study developed protocols and procedures for identifying and coding instances of deviance/conflict from written records of space missions and polar expeditions. The methodology is reported in Dudley-Rowley $(1997,2000)$. Multiple coders were then able to produce reliable data for a stratified random sample of crews in extreme environments. Three space missions were examined: Apollo 11 (Aldrin 1973), Apollo 13 (Lovell and Kluger 1994), and Salyut 7 (Lebedev 1988). Four Antarctic expeditions were examined: the western party field trip of the Terra Nova Expedition (Back 1992), an International Geophysical Year (IGY) traverse (Pirrit 1967), the Frozen Sea (Lewis and George 1987) and the International Trans-Antarctica (Steger) (Steger and Bowermaster 1992) expeditions. Three Arctic expeditions were examined: the Lady Franklin Bay (Greely 1886), Wrangel Island (Stefansson 1925), and Dominion Explorers' (Pearce 1930) expeditions. Coders quite consistently identified the same events and incidents as evidencing deviance/conflict.

Multiple rounds of reliability testing prior to the coding process had determined that incidents of deviance, conflict, and dysfunction were characterized by three types of actions and events:

1. Actions and events stemming from mental disorders;

2. Actions and events involving physical violence and verbal abuse; and
3. A broad area of actions and events like hoarding resources, deliberately breaking or not maintaining equipment needed for survival, leaders failing to lead, etc.

\section{THEORETICAL BACKGROUND}

The study of these data was informed by the theoretical work of Peter Blau and Bruce Mayhew. Peter Blau outlined a relatively formalized theory of the effects of social structure on rates of intergroup association (1977). $\mathrm{He}$ thought that rates of intergroup association were essential determinants of social solidarity and social cohesiveness. Blau's primary concern was to identify factors that either fostered or hindered contact and interaction among people who occupied different positions in a multidimensional social structure.

For Blau's purposes, the assumption, and oft-observed empirical regularity, that, ceteris paribus, people tend to prefer to interact with others who are socially similar -homophily -- is a largely unexamined predicate for his exploration of how, despite this in-group preference or bias, differing distributions of people across social dimensions will, by chance alone, promote different rates of intergroup association. For example, if there are two socially distinguishable groups (e.g., males and females) -- a 50:50 distribution will, under the same constraints, promote more intergroup association than a 10:90, or 20:80 distribution.

For our purposes, it is worth examining this assumption more closely. Why homophily? The simplest and most straightforward reasons are: time and energy. Other things being equal, communication among people who are culturally and socially similar is quick, and less likely to produce misunderstanding and inadvertent offense than it is among those who are different. Think of a continuum of communication (Figure 1). On one end lies attempted communication among people who speak mutually unintelligible languages and who differently interpret the same physical gestures (e.g., head nodding or hand gestures). On the opposite end is the communication of individuals who are so similar in experiences, education, and familiarity that they can complete each other's sentences before they are spoken. In general, communication among those on that end of the continuum will be quick and efficient. Communication among those on the former will be difficult, time consuming, frustrating, and fraught with dangers of inadvertent insult and misunderstanding.

Figure 1: A Continuum of Communication

\section{$\leftarrow-$}

Attempted communication among those who speak mutually unintelligible languages and interpret physical gestures differently (difficult)
Communication among those who are so similar in language and backgrounds that they can complete each other's sentences (easy) 
Therefore, although we sometimes enjoy communicating with people of different backgrounds and culture, because it is stimulating and interesting, we typically welcome the return to less problematic communication with people who "understand us."

\section{HYPOTHESES}

The investigators coupled Blau's train of thought with the insights of Bruce Mayhew. Mayhew asserted that contacts and conflicts are likely to increase geometrically with increasing group size (Mayhew and Levinger 1976), and become more likely over time. From these twin assumptions, the authors could derive some a priori hypotheses about conflict among the crews of space missions and polar expeditions. These are that:

1. Heterogeneous crews with respect to nationality, sex, age, and experience will have higher rates of deviance and conflict than homogenous crews.

2. Larger crews will have higher rates of deviance and conflict than smaller crews.

3. Rates of deviance and conflict will increase with increasing mission duration.
Finally, based on anecdotal accounts, the authors could hypothesize that rates of deviance/conflict will vary systematically by mission interval. More specifically that:

4. The rate of deviance will peak in the third quarter.

The latter hypothesis is the classic assumption of the anecdotally based "third-quarter phenomenon", that a crew will demonstrate a high degree of deviance, conflict, and dysfunctionality after the midpoint of the mission.

\section{MEASURES}

To enable comparisons across cases with differing size crews and of differing duration, standardized rates of deviance were computed for each quarter of the missions/expeditions. This computation was made by dividing the total number of coded dysfunctional events in a quarter by average crew size (in a number of cases people left and joined crews during missions/expeditions). The computation was completed by then dividing by the quarter's duration in days. To make the numbers more manageable, this rate was multiplied by 1,000. The figures reported for quarterly rates of deviance/conflict, therefore, are per crew member per 1,000 days. Rates for the complete missions/expeditions were computed by taking the simple average of its rates for four quarters (Table 1 ).

Table 1: Data From Ten Missions and Expeditions

\begin{tabular}{|c|c|c|c|c|c|c|c|c|c|}
\hline Mis. & $\begin{array}{l}\text { Tot./Q } \\
\text { Dur. }\end{array}$ & AvCrw & SexHet & NatHet & AgeRn & $\begin{array}{l}\text { Exp } \\
\text { Het }\end{array}$ & $\begin{array}{l}\text { Dev } \\
\text { Acts }\end{array}$ & $\begin{array}{l}\text { DaPr } \\
\text { Rate }\end{array}$ & $\mathbf{x 1 0 0 0}$ \\
\hline Frozen & $480 / 120$ & 5.88 & 0.45 & 0.67 & 40 & 0.43 & 13.13 & 0.02 & 20 \\
\hline Salyut 7 & $212 / 53$ & 2.75 & 0.05 & 0.49 & 2 & 0 & 8.13 & 0.06 & 60 \\
\hline Wrangel & $720 / 180$ & 3.75 & 0.33 & 0.48 & 10 & 0.33 & 20.63 & 0.03 & 30 \\
\hline LFB & $\begin{array}{l}1080 / \\
270\end{array}$ & 23.13 & 0 & 0.22 & 23.75 & 0.23 & 41.63 & 0.01 & 10 \\
\hline Steger & $224 / 56$ & 6 & 0 & 0.82 & 10 & 0 & 5.25 & 0.02 & 20 \\
\hline TerNov & $48 / 12$ & 4 & 0 & 0.63 & 11 & 0.38 & 0.5 & 0.01 & 10 \\
\hline DomEx & $72 / 18$ & 16 & 0.41 & 0.4 & 60 & 0 & 2.25 & 0.01 & 10 \\
\hline IGY & $88 / 22$ & 8.88 & 0 & 0.63 & 20 & 0.2 & 0.5 & 0 & 0 \\
\hline Apollo11 & $8 / 2$ & 2.75 & 0 & 0 & 0 & 0 & 0.38 & 0.08 & 80 \\
\hline Apollo13 & $6 / 1.5$ & 3 & 0 & 0 & 2 & 0 & 1 & 0.22 & 220 \\
\hline
\end{tabular}

NOTE.-Depicted are total and quarterly duration of mission in days, average crew size, average sex, nationality, age, and experience heterogeneity, average number of deviant acts, rate of deviance in terms of deviant acts/days/number of persons (DaPrRate), and the simple average of each mission's deviance rates for four quarters per crew member per 1000 days $(x 1000)$. 
Sex, nationality, and experience heterogeneity were calculated in accordance with "Blau's H." Blau's H is a standard probability computation. It is made by subtracting the sum of the squared proportions in each category of heterogeneity from 1 (Blau 1977). Intuitively, it is the probability that two randomly selected crewmembers will belong to a different category of the dimension of heterogeneity in question. In this study, age heterogeneity was specified by the span between eldest and youngest members of missions.

\section{EMPIRICAL RESULTS}

Every one of the a priori hypotheses was challenged by the data analyzed in the pilot study. Contrary to the hypotheses:

1. heterogeneous crews had lower rates of deviance and conflict (Figure 2),

2. larger crews had lower rates of deviance and conflict (Figure 3), and

3. deviance and conflict tended to decline with increasing length of mission (Figure 4).
It is also noteworthy that rates of deviance/conflict were higher in the space missions than they were in the polar expeditions. Salyut 7, which had the lowest rate for the space missions, had twice the rate of deviance/conflict as the highest polar expedition, Wrangel Island. One possible explanation is that there may be beneficial ranges along a continuum for the expression of conflict (Figure 5). Somewhat analogous to marriage, it may be the case that crews that never express open disagreement, or those who fight "like cats and dogs," do not function as well as those who confront and constructively work out openly expressed disagreements. Anecdotally, American space crews are well-known for suppressing the expression of conflict. The polar expeditions which had the most severely expressed conflicts in the sample were Frozen Sea, Wrangel Island, and Steger expeditions. They demonstrated the highest rates of deviance/conflict compared to the other polar expeditions (Lady Franklin Bay, the Terra Nova field party, Dominion Explorers', and the IGY traverse). Only in the final quarter of the Lady Franklin Bay expedition, when the men were mentally deteriorating from starvation did severely expressed events occur. It was during that quarter that their rate of deviance, conflict, and dysfunction rose sharply. Of the space crews in this sample, the Salyut 7 crew more openly expressed more conflict among themselves than both Apollo crews did. Salyut 7 had lower rates of deviance/conflict than those crews.

Figure 2: Average Deviance Rates by Quarters for Crews Heterogeneous and Homogenous for Sex, Nationality, Age, and Experience

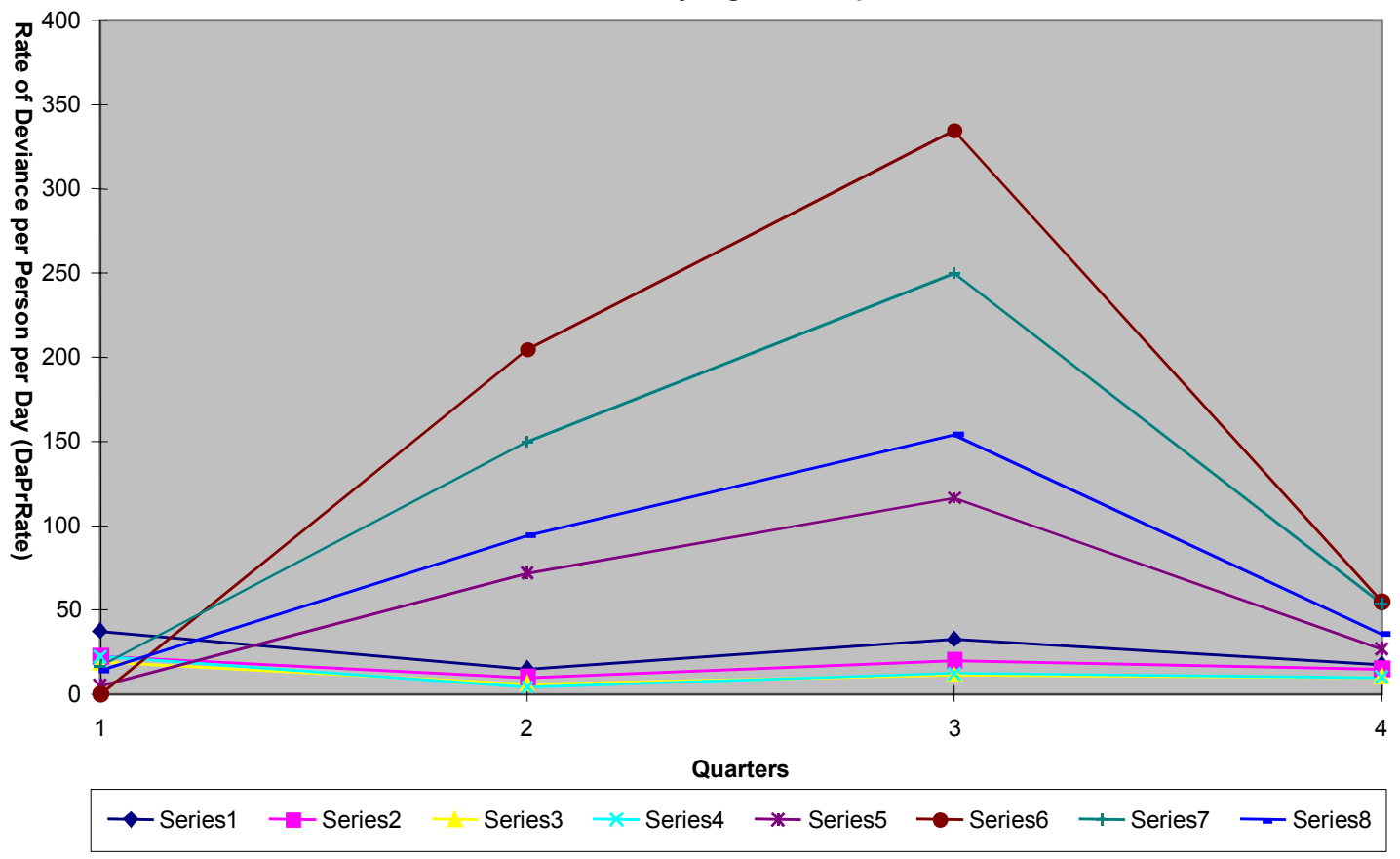

Series from L-R: 1 . sex heterogeneity; 2. nationality heterogeneity; 3 . age heterogeneity; 4 . experience heterogeneity; 5. sex homogeneity; 6 . nationality homogeneity; 7 . age homogeneity; 8. experience homogeneity 
Figure 3: Rate of Deviance by Average Crew Size

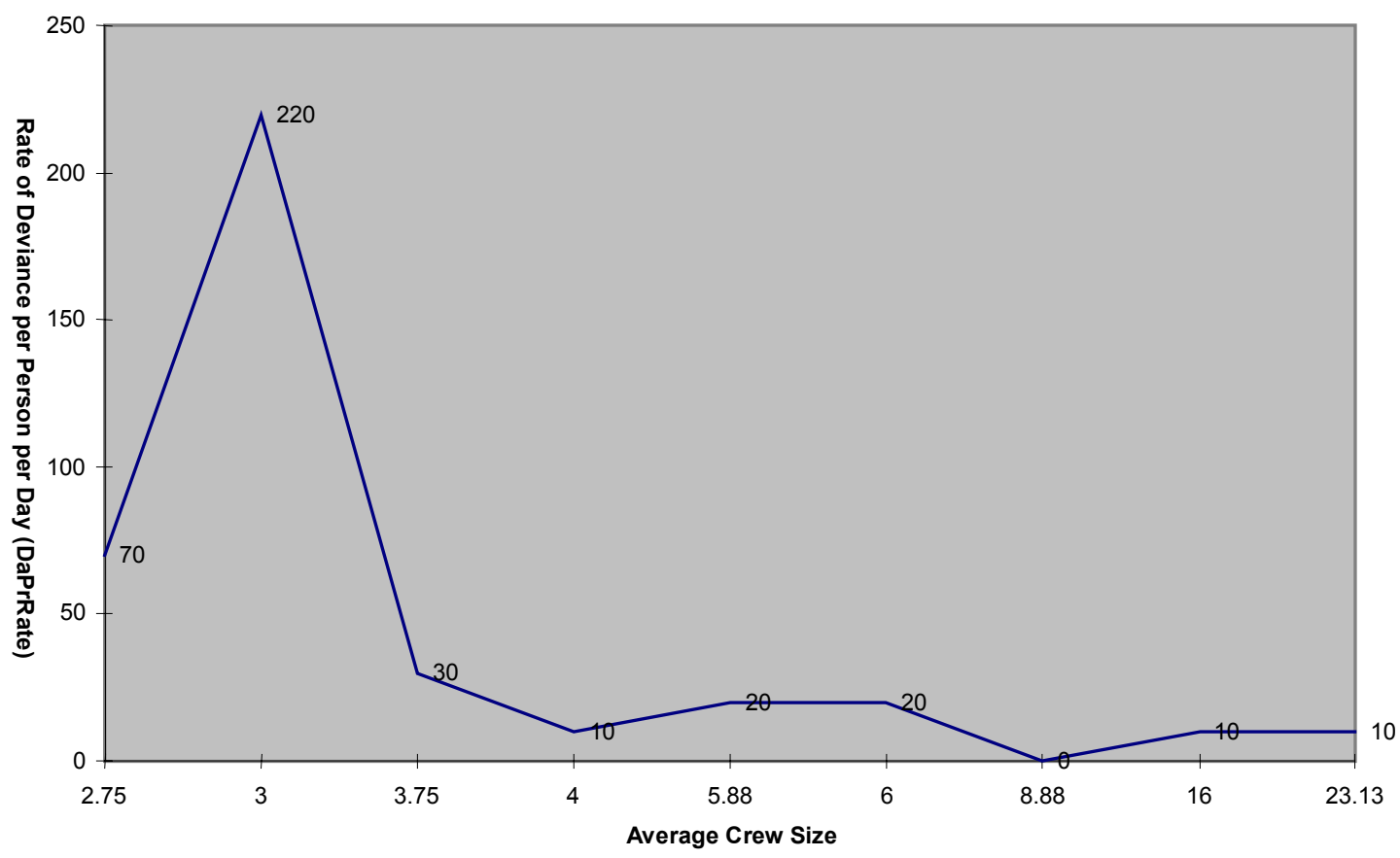

NOTE.-- DaPrRate = Deviant acts/days/no. of persons. L-R: Apollo 11 (averaged with Salyut 7),Apollo 13, Wrangel, Ter. Nov. , Frozen Sea, Steger, IGY, DomEx, LFB

Figure 4: Rate of Deviance by Mission Duration

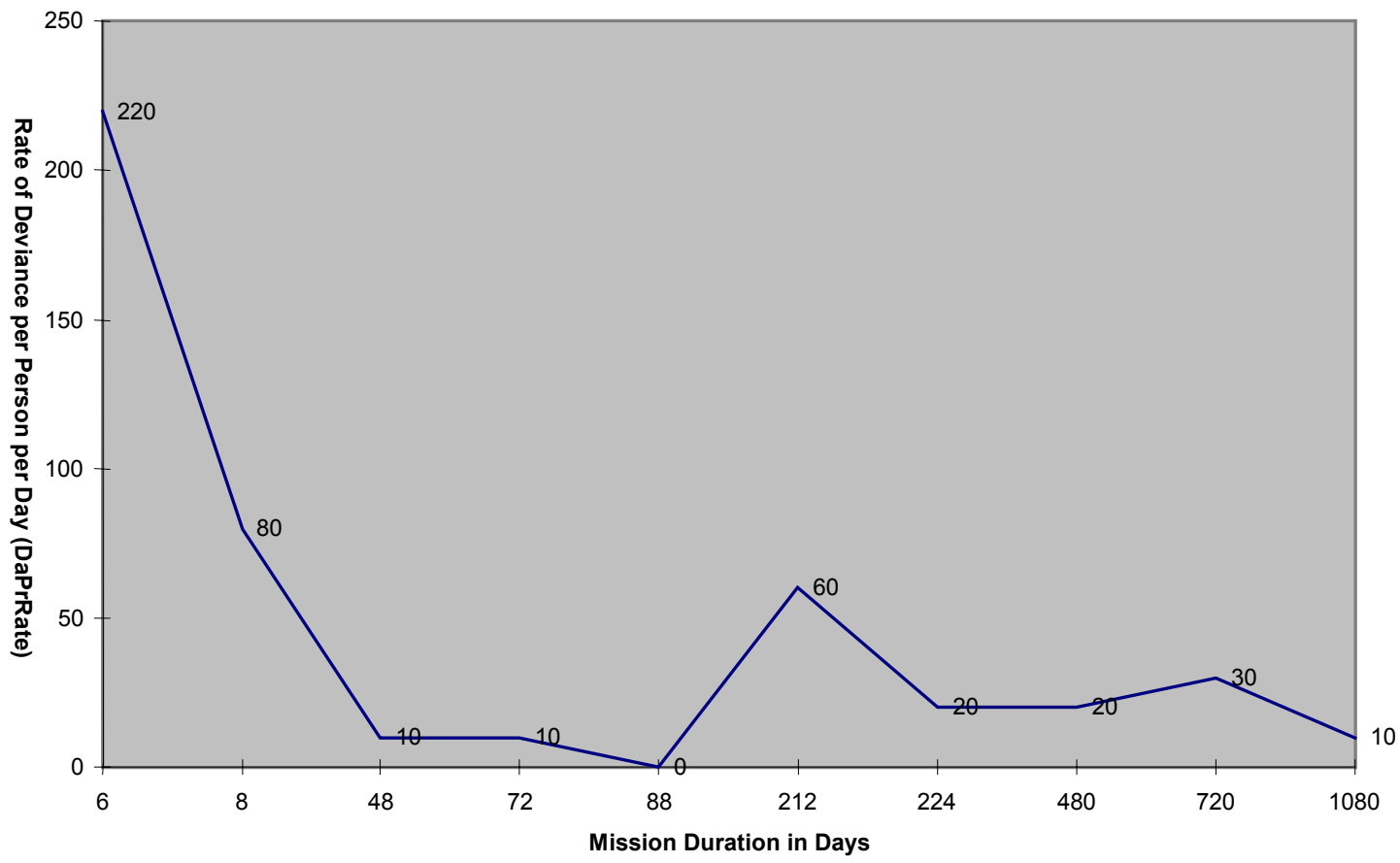

NOTE.-- DaPrRate = Deviant acts/days/no. of persons. L-R: LFB, Wrangel, Frozen Sea, DomEx, Ter. Nov. , Apollo 11, Apollo 13 
Figure 5: A Continuum for Expression of Conflict

Dysfunctional Functional Dysfunctional

$\begin{array}{ll}\text { People who fight like“cats } & \begin{array}{l}\text { People who openly express } \\ \text { and work out }\end{array}\end{array}$

disagreements

The "third-quarter phenomenon" was demonstrated over the 10 cases treated when their third-quarter deviance rates were averaged (Figure 6). Further examination showed, however, that it was only clearly present in Apollo 13 and Salyut 7. Their third quarters contributed to the placement of the tall peak in this graph where the third quarters across the sample are averaged. Several issues are raised by this specification. Perhaps it indicates that space missions are different from polar expeditions in this regard. However, it is likely that some other factor is at work. Except for Apollo 11, all the crews in the sample had some degree of heterogeneity in composition. The space crews had the least heterogeneity of all. The result may indicate that the third-quarter phenomenon only occurs in relatively homogenous crews. This might help explain its "anecdotal" status; researchers studying more homogenous crews find it while those working with more heterogeneous crews do not. That there was no thirdquarter peak during the Apollo 11 mission detracts from this line of speculation. However, it could be that the high degree of uniqueness of this mission had a leveling effect in its third quarter. The deviance that did appear during that mission occurred in the second quarter, during the most delicate part of that mission, making such an inference attractive.

Apollo 11 and Apollo 13 were very brief missions and investigators wondered if the technique of extrapolating rates from very short time frames might have been a source of bias. In other words, was the Apollo 13 thirdquarter peak distorted out of proportion by a method of computation that might be biased when used over short time frames? However, both the mathematics and the expeditionary record suggest that a few dysfunctional actions and events over a short period can be as remarkable as many such actions and events over the course of a longer mission. It may be that pressures to complete assigned tasks on short schedules may contribute to the effect. The fact that a third-quarter peak was found during the Salyut 7 mission, a relatively long space mission, one where the schedule resembled more of a daily round on the ground, lends that idea credence.

Figure 6: Rate of Deviance by Mission Quarter

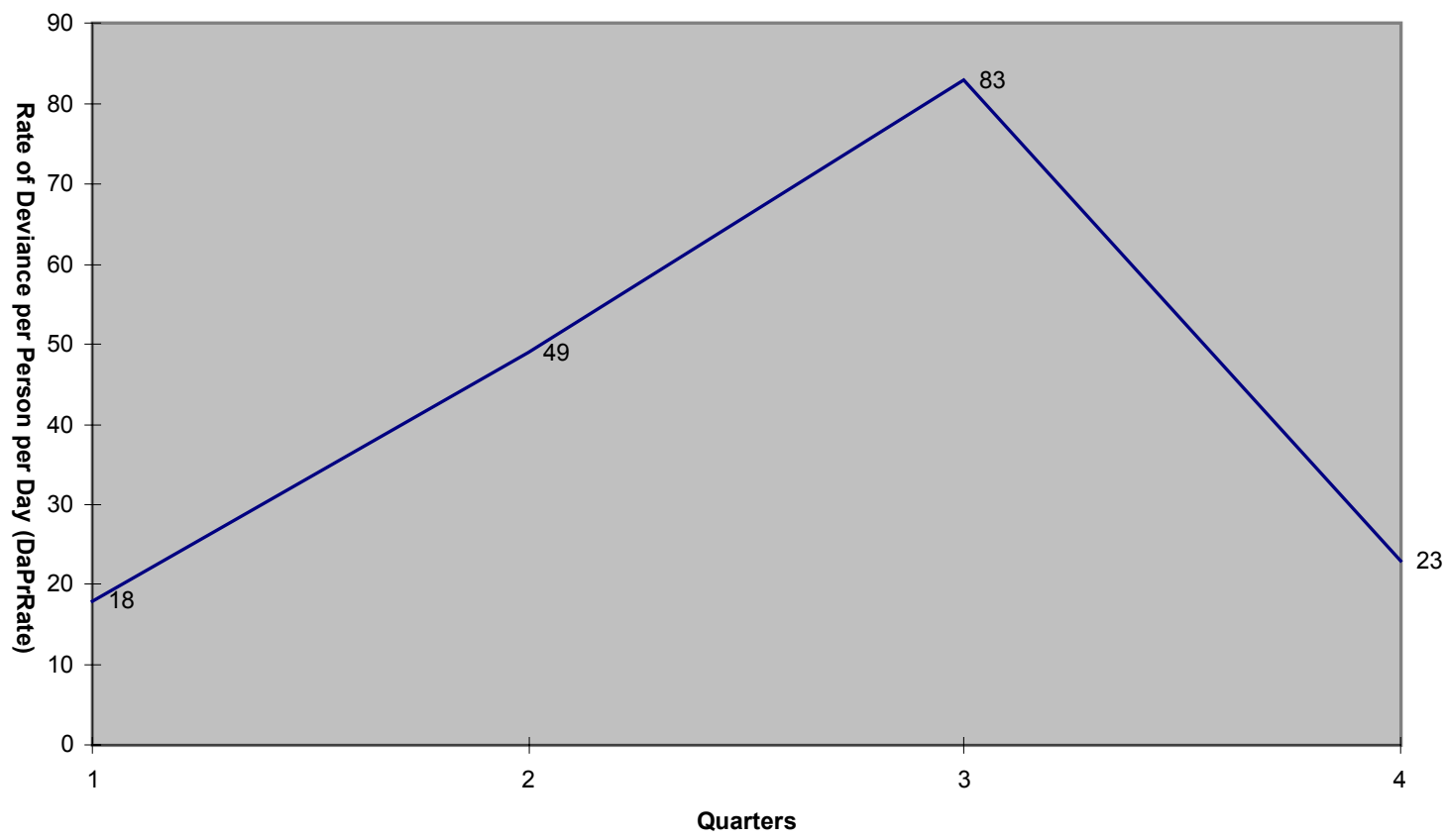

NOTE.-- DaPrRate $=$ Deviant acts/days/no. of persons 


\section{DISCUSSION AND CONCLUSION OF THE PILOT STUDY}

Three accomplishments of the pilot study are worth emphasizing. First, it documented that the protocols and coder training developed for the study did indeed produce strong coder agreement (intercoder reliability) on instances of deviance/conflict from mission and expedition narratives. Second, standardized rates of deviance displayed distinct and intriguing patterns across different crew profiles and space and polar settings. The most important finding, however, was that homogenous crews appear to start out with low rates of deviance/conflict that tend to dramatically increase over the course of the mission, while heterogeneous crews have initially high rates of deviance/conflict that decline over the course of the mission. This may indicate that homogenous crews (falsely) believe that they have no interpersonal issues or conflicts, or suppress the expression of them, until they are too intense to ignore, while heterogeneous crews expect and acknowledge them at the outset. It may also indicate that members of heterogeneous crews may be better able to find distinct and comfortable "niches," than can members of homogenous crews who are so much alike.

The larger study features longer space missions and shorter polar expeditions and more heterogeneous space missions and more homogenous polar expeditions. It will be interesting to see if the larger, more diverse, sample will support the distinctive patterns established by the pilot study.

\section{INTRODUCTION TO THE SECONDARY ANALYSIS}

Traditionally, long-duration missions in extreme environments have been understood to encompass missions of some undetermined chronological length that is longer than "short" duration. While a general consensus exists that two weeks fall within the "short" duration range, there is decreasing consensus on the threshold that qualifies a mission for "long-duration" status. Classifications based on space missions have a clear break between the 14-16 day shuttle missions to the months-long rotations aboard Skylab, the Salyut stations, and Mir with very little in between. Even earlier space missions lasted from a few hours to a few days in duration. Analog and simulation studies have ranged from 2 weeks to 10 months. Actual polar expeditions, especially the earlier ones, were in the field for several years.

Efforts to find patterns in performance, cognition, and physical and mental well-being which correspond with short- and long-duration mission profiles have not found clear differences. Decrements in performance, cognition, and group dynamics have manifested on missions as short as two weeks. But does mission duration alone dichotomize missions? There may not exist key elements linked to actual real-time duration that require different preparation, support, and training for longduration missions compared to short-duration missions. Perhaps the key elements we are looking for are linked to those things which "alter" subjective time for extreme environment crews and which make the mission in the extreme environment situationally different from the baseline societies from which team members derive.

The concern with subjective time and similar factors could be couched as a class of "situation awareness" problem. In the literature of human factors, the problem of situation awareness typically addresses how conscious the operator of an aircraft or other vehicle is of outside conditions or the fitness of his/her craft (e.g., attitude of a transport plane) (Endsley and Bolstad 1994). In a usual situation awareness problem, a person watching from the ground might observe that an aircraft "was flying upside down." Radio contact with the cockpit crew might confirm that the operators of the plane were not aware of that. The problem of subjective time and other situational factors emerging from the interplay of the extreme environment group, its individual members, and its environment is a bit more insidious for the observer. Outside observers whose business it is to be concerned with space and other extreme environment crews, like mission controllers, are not traditionally trained to recognize that "what is real" for a person or a group away from their larger baseline group can differentiate -- and differentiate quite rapidly -- from "what is real" for the baseline. In examining the expeditionary record, it is obvious that outside observers from baseline societies have had difficulties adequately assessing the situations of extreme environment teams, which become microsocieties in themselves.

Outside observers can be successful in targeting problems of basic quantity (e.g., the crew will run out of breathable air in two hours time.) But, observers and controllers are likely to be totally oblivious to a wholly different metric which deals with things of a more social psychological nature. A hypothetical example illustrates this point: a projects supervisor at an Antarctic winterover base, over a three-month period, committed 3,030 "microaggressions" against his teammates numbering 31 persons. His superiors, elsewhere in the world, wonder why the team cannot keep pace with its duties, and why more people than before have reported a higher than average number of ailments ranging from recurring headaches and stomach problems to depression (after Pierce 1991). If the psychosocial dimension would become a greater concern among agencies that employ extreme environment workers, it would produce useful information for the areas of crew selection, training, and mission planning and policy. The importance of the psychosocial dimension and the crew's definition of the situation are things that astute and specially empathetic Capcoms have qualitatively known about for years, as have teams which routinely engage in the rescue of hostages or people in trouble in extreme environments. 
Factors of a psychosocial nature have been at work in recent studies preparing for the operation of the International Space Station. While it may be documented that a woman serving aboard a Russian space station simulator for some months in 1999 and 2000, was twice subjected to physically aggressive sexual advances in the simulation by a male coparticipant (CBC News 2000, Guly 2000), it is reductionist to assume that is all that happened (Sandal 2000). The crew's situation up till the time of these events had taken on a reality that had differentiated from the reality experienced by the baseline outside of their chambers. The differentiation process began the moment each participant stepped into the simulator. Actions, gestures, settings, materials, and words would have come to have special meaning for the participants. They formed a microsociety which began to change from those different cultures from which they derived (Bishop 2000), even in light of personal and prior cultural differences. The woman participant found the kisses intrusive, threatening, and a prelude to rape. She felt a pervasive fear of being assaulted for days after the overtures. It is fair to say that inside the Russian simulator, a kiss was not just a kiss.

What goes on in the psychosocial dimension can work for good or ill. Microsociety formation is also at the heart of the successes in functioning of the Mir-NASA crews in spite of different national agendas. One thing that stood out in the more problematic of these flights is the differential in the timing of task performance and the quantity of tasks scheduled. The Russian space station was set up with a specific work and living cycle in mind that resembled more of a workday on the ground, and its focus was on missions of real-time longer duration.

Enter the American astronaut, trained to do a grueling round of scientific and commercial work within a shorter period. But Mir is not set up for it, nor are the Russians compatibly trained or even predisposed to assist. After a while, it occurs to the astronaut that equipment is not where he can put his hands on it quickly, he is getting overworked and off-track. He cannot eat or sleep at regular times. He is attempting to follow a schedule that is an interminable expansion of a short shuttle mission. He does not have a standard operating procedure to look up what to do in this situation. His Russian fellows have duties and problems of their own keeping the station up and running and cannot help him catch up. The American feels let down, undermined, and betrayed.

In turn, his Russian fellows wonder what is the matter with him? Is he an idiot? The American and his Russian fellows have some heated discussion about their problems with one another and it dawns that they are trying to track along two different schedules from two different national foci. Afterwards, they do the best they can, under the circumstances, to merge their interests and schedules (Burrough 1998).
Attention to the psychosocial human factors and understanding how situations in the extreme environment can deviate from the baseline on the ground or "back home" can lead to the optimization of the objectives of space missions which will become longer and more autonomous. In this vein, it is as important an area of research as are radiation exposure, bone demineralization, and cardiovascular studies.

\section{METHODS}

The secondary analysis began with an insightful question, "Are three months aboard the International Space Station (ISS) really as 'long-duration' as compared to three months outbound to Mars?" The pilot study, described above, offered compelling data to answer this question. A secondary analysis of the sample set was done. The investigators content-analyzed for other factors which might have distinguished the missions from one another, besides the environment, crew size, heterogeneity, mission duration, and mission interval which the original study examined. Several items emerged which increased the complexity and danger of missions. Indeed, the notion of duration seemed to change as missions became more complex and dangerous in terms of increasing separation from various aspects of individuals' normal baseline routines (e.g., home, the training environment, etc.) and with the introduction of different routines shaped by the expeditionary social and physical environments. The hypothesis emerged: As the crew's control over its environment decreases, its members' subjective experience of the passage of time and the situation increasingly differs from the point-of-view of its baseline. A vernacular example of what we mean comes from a popular song. Gordon Lightfoot, in singing about the wreck of the Edmund Fitzgerald on Lake Superior during a freak storm, asks, "Does anyone know where the love of God goes when the waves turn the minutes into hours?" Several anecdotally recorded phenomena are likely related: wanton massacres of civilians in ground combat environments by small groups of military personnel who have been trained in such values as "duty and honor"; missionaries "going native"; reports from accident victims who recall how time seemed to slow as their accidents unfolded, etc. Some of the topics studied by those who examine human performance in extreme environments are likely related, like the phenomenon of perceptual narrowing (Baddeley 1972).

The stratified random sample of Arctic and Antarctic expeditions and space missions in the pilot study was reanalyzed. The Apollo missions to the moon ranged from 6-8 days, and the Salyut 7 orbital mission lasted over 200 days. The Lady Franklin Bay and Wrangel Island expeditions to the Arctic were the longest missions at 1080 and 720 days respectively. The Frozen Sea and Steger's expeditions were 480 and 224 days respectively. The IGY field traverse, the Dominion 
Explorers' Expedition, and the Terra Nova western party field trip were 88,72 , and 48 days respectively.

Seven factors emerged which seemed to coincide with the subjectivization of time and the differentiation of situational reality for the crews from baseline. These were:

1. increasing distance away from rescue in case of emergency (lessening chances of "returnability");

2. increasing proximity to unknown or little-understood phenomena (which could include increasing distance from Earth);

3. increasing reliance on a limited contained environment (where a breach of environmental seals means death or where a fire inside could rapidly replace atmosphere with toxins);

4. increasing difficulties in communication with Ground or Base;

5. increasing reliance on a group of companions who come to comprise a microsociety as time, confinement, and distance leave the larger society behind, and where innovative norms may emerge in response to the new sociophysical environment;

6. increasing autonomy from Ground's or Base's technological aid or advice; and

7. diminishing resources needed for life and the enjoyment of life.

The presence and prevalence of these factors in each of the sample missions/expeditions are discussed in Dudley-Rowley et al. (2001).

\section{RESULTS, DISCUSSION, AND CONCLUSIONS OF THE SECONDARY ANALYSIS}

It is widely known in the study of disasters and hazards, both natural and human-caused, that the subjectivization of time and other situational factors are experienced differently from baseline. One expects the most severe disasters to generate the greatest differential in the reality of the situation from baseline. By analogy, one might expect extreme environment missions and expeditions of most severity or greatest risk to generate the greatest differential in the reality of the situation from baseline. If we were to rank the missions and expeditions in this sample according to this line of reasoning, we might expect to see the greatest difference from baseline in the following groups, ranked on order of greatest differences to fewest ones:
1. Space and polar missions where there were deaths and disasters (where risk was demonstrated).

2. Space missions where deaths and disasters did not occur (but where there was risk in the certain knowledge that something could happen to systems upon which life depended).

3. Early polar missions (where much was unknown about the Arctic and Antarctic, and where was unavailable the level of technology that would have alleviated risk and would have made working and living in these regions more like baseline).

4. Modern polar expeditions (where technology has done much to alleviate risk and make living and working in these regions similar to baseline).

The missions and expeditions were ranked by prevalence of the seven factors which might correspond with the differentiation in the subjectivization of the passage of time and in the situational reality for the crews from baseline. From high to low, the ranking revealed: Lady Franklin Bay (7); Wrangel Island, Apollo 13 (6); Salyut 7 (5); Terra Nova, Apollo 11 (4); Dominion Explorers' (3); Frozen Sea (2); IGY (1); Steger (0).

This ranking is quite consistent with the ranking generated from the disaster analogy. The Lady Franklin Bay Expedition suffered 18 deaths of its complement of 25 , and the rest were starving when found. The Wrangel Island Expedition suffered four deaths out of its crew of five. Apollo 13 was a catastrophe that was remarkable in its recovery of the crew intact. The Salyut 7 mission, the Terra Nova western field party, and the Apollo 11 mission all had a high degree of risk. The later polar expeditions rank below these missions.

All the space missions and the earliest polar expeditions are above or hover just below the median (3.5). Although this sample is too small to say anything definitive, there is something suggestive in the idea that there are similarities in space and early polar exploration in how humans subjectify mission/expedition duration or in how their situational reality deviates from baseline. Compellingly, deviation from baseline in experience of time and situational definition occurred in the Lady Franklin Bay and Wrangel Island expeditions, Apollo 11, Apollo 13, Salyut 7 missions, and perhaps arguably, to a small degree in the Dominion Explorers' Expedition. The Terra Nova field party, just above the median, did not record any differential in the way its crew subjectivized time or defined the situation from their base camp. Even so, these results suggest that as extreme environment crews' control over their environment decreases, their subjective experiences of time and the situation increasingly differ from their baselines (Table 2). 
THE SEVEN FACTORS OF TIME SUBJECTIVIZATION AND SITUATIONAL REALITY

Factors 1 and 2: increasing distance from rescue and increasing distance from Earth (or increasing proximity to unknown phenomena) co-occurred six out of eight times one or the other occurred. They co-occurred in all the six expeditions above the median. This may suggest that it is hard to plan and provide rescue when there are a critical number of unknowns.

Factor 3 (reliance on a contained environment where breach of environmental seals might result in death) occurred in all the space missions, of course, but also occurred in two of the seven polar expeditions, an older Arctic expedition and a modern Antarctic expedition.

Factor 4 (increasing difficulties in communication with Ground or Base) occurred four times. This factor played a major role in the difficulties or demise of three of four of those expeditions (Lady Franklin Bay, Wrangel Island, and Dominion Explorers').

Factor 5 (increasing reliance on a group of companions who form a microsociety over time) occurred five out of 10 expeditions/missions. Each time, microsocial innovations occurred as a means to handle stress or pressure from within the group or directly associated with the field expediency of the situation.

Factor 6 (autonomy from Ground's or Base's technological aid or advice [whether or not it was needed]) occurred seven out of the 10 cases. Where autonomy does not occur is in the more modern Antarctic expeditions. All the missions/expeditions above and around the median saw some degree of autonomy from Ground Control or Base as compared to these.

Factor 7 (diminishing resources threatening to life or enjoyment of life) occurred three out of the 10 cases (Lady Franklin Bay, Wrangel Island, and Apollo 13). The Lady Franklin Bay Expedition's numbers were whittled down directly as a consequence of this, the Wrangel Island Expedition suffered a slightly more deadly result as a consequence of this, and Apollo 13's mission objectives took on a far different parameter when resources necessary for life diminished rapidly.

Proximity to the unknown, autonomy, distance, and microsociety formation were present in every one of the expeditions where there was deviation from baseline in experience of time and situational definition. This content analysis suggests that these factors in combination contribute to increasing these differentials.

\section{IMPLICATIONS FOR HABITAT AND WORKPLACE DESIGN IN EXTREME ENVIRONMENTS AND CONCLUSION}

This pilot study implies several design specifications for habitats and workplaces in extreme environments.

Crew size. Overall, larger crews were less dysfunctional than smaller ones. The crew which demonstrated the least deviance, conflict, and dysfunction of all was one that numbered about nine persons. These findings imply that habitat and workplace designers plan for larger facilities in extreme environments, such as those anticipated for Mars operations.

Heterogeneity. Any kind of heterogeneity studied herein seemed to be beneficial. Heterogeneity distinguishes people from one another, which makes them interesting to each another over the long haul and which offers complementary experiences and skills in order to allow the group to arrive at useful, innovative solutions during the expedition or mission. This most likely extends to other kinds of heterogeneity beyond the demographic sort (e.g., sex, nationality, and age). There is evidence that psychological heterogeneity is beneficial to the extreme environment crew as well (Weed 2001). This finding implies that extreme environment facilities be designed to accommodate a heterogeneous group of users.

Mission Duration. The longer missions in this study had lower rates of deviance, conflict, and dysfunction. Longer missions provide for a daily round of activities that resemble something more akin to an everyday, ordinary schedule. People have time to socialize and to get to know each other's strengths and weaknesses over a longer period. In the missions studied, even the presence of people with mental disorders and extreme personalities were tolerated by members of the longer expeditions to greater or lesser degree. Even in the expedition where a man was executed (the analog of a troublesome astronaut being shoved out of an airlock), his behaviors were taken in stride until the crew's situation changed to make his behaviors fatal to his mates rather than simply bothersome.

The implication for design from this finding is to construct living and workspaces that can be used for years, if need be, and which promote a daily round of activities that resemble ordinary life.

Mission Interval. This study demonstrated dramatically that there are two distinctive patterns over mission intervals that are linked to heterogeneity and homogeneity of crews. More heterogeneous crews start out with a level of deviance, conflict, and dysfunction 
suggesting that they have some trouble coming to terms with their differences at the outset. However, they do come to terms with those differences and make use of the benefits of the heterogeneity that they possess. Their rates of dysfunction drop. More homogenous crews are different. Their deviance, conflict, and dysfunction rise sharply after the mid-point of the mission. They are likely working well together at the outset, as would be expected of people with similar training and background. However, their similarities become tiresome after a while. Where none are available who have different life and work experiences, they may find themselves in situations where they cannot "think outside of the box" in a dilemma. The steep gradients between the third and fourth quarters of the missions of homogeneous crews suggest that they suffer much in attempting to reduce their dysfunction and achieve their mission objectives before end of mission.

This finding implies that heterogeneity is not only very important in crew composition, but in design. It will be beneficial for facilities to be designed in such a way as not to "level" group heterogeneity. An example of this from submarine experience is to provide personalized sleeping accommodations to each crewmember, i.e., no "hot bunking". Homogeneity can be lessened through variety in surroundings within the limitations of confinement - the use of different colors, textures, and architectural features that allow for favorite items or spaces and personal identification.

Time Subjectivization and Shift in Situation Reality From Baseline. The issues of distance from rescue, proximity to the unknown, reliance on a limited contained environment, difficulties in communication, microsociety formation, increasing autonomy, and diminishing resources will be the greatest challenges that designers face for extreme environments, especially for a Mars mission. However, innovations in habitat and workplace design can reduce the differentials that emerge between crews and baseline. Many of these innovations can find their inspiration by looking to what has already been learned from the expeditionary experience on the seas, at the poles, and in space (Stuster 1996).

The distance between the field crew and baseline can be perceptually minimized by providing in situ reminders of the baseline environment. Little things count for much. For example, Russian designers used wood trimmings in their prototypical Mars habitat based on experiences of cosmonauts who delighted in things that reminded them of terrestrial life - the taste of a fresh onion or watching a plant grow. Many of the other challenges can be met by designing for ease of engagement of the field environment that will allow "unknowns" to become known and make a limited contained environment seem less confining. The Fram, an Arctic expeditionary ship of the late 1800s, was highly successful as an extreme environment habitat and workspace because its designer, Fridtjof Nansen, addressed the seven factors discussed here. He studied the accounts of previous expeditions, and he specially designed the Fram from the ground up using what information he could glean on the movement of polar pack ice, a little-known phenomenon at the time (Stuster 1996: 300-301). The way the ship responded to being frozen into the polar pack and its course in the northern polar regions confirmed the movements of the permanent pack. As a result, the Fram was much more than a utilitarian component of exploration, it was the hub of a thriving microsociety, autonomous from the rest of the world, with its crew returning relatively unscathed after more than three years in the Arctic.

The difficulties in communication between Earth and Mars are shaped by physical laws. There is no way to get around the problem of time delay in reception between points. However, enhanced communication satellite links and data compression technology might come to relieve some of the dangers that append to that problem. In the meantime, habitat and workspace design that promotes successful autonomy of the mission and that gives the crew a security in self-reliance and a measure of comfort being "on their own" can minimize the need to communicate. Any systems that make use of in situ resources will be beneficial. However, a warning is called for here. Care must be taken in assuming too much about availability and acquisition of in situ resources. The example of Arctic exploration has been held up as the "poster child" of in situ resource utilization on Mars (Zubrin and Wagner 1996). But, as close study of the expeditionary record shows, assumptions about availability of resources and successful acquisition techniques based on experiences in one part of the Arctic did not transfer to other parts of the Arctic. A number of Arctic expeditions came to grief because of these assumptions. Sometimes climatic conditions simply changed and the same spot in the Arctic became environmentally different some years hence, as in the case of the changeable nature of semi-permanent pack ice. Similar problems obtained in trying to import Arctic techniques to the Antarctic. The Martian landscape may well be a polar desert, but it is a polar desert on another planet.

The results and implications of this quantitative study came from a small sample. Overall, its results and implications were not inconsistent with findings of qualitative studies over a greater number of cases or from studies that examined one simulation or one mission (Evans, Stokols, and Carrere 1988). These studies all end on the same note: psychosocial human factors play a role in how well or how poorly future space crews carry out their mission objectives. 
Table 2: Differentiation Between Crews and Baseline Over Ten Missions/Expeditions in Terms of Factors of Time Subjectivization and Situational Reality

\begin{tabular}{|c|c|c|c|c|c|c|c|}
\hline Missions & 1 & 2 & 3 & 4 & 5 & 6 & 7 \\
\hline & Distance & Unknowns & Contained & Commo & Microsoc. & Autonomy & Resources \\
\hline LFB & $*$ & $*$ & $*$ & $*$ & $*$ & $*$ & $*$ \\
\hline Wrangel & * & * & & $*$ & * & * & * \\
\hline Apollo 13 & $*$ & $*$ & * & & * & * & * \\
\hline Salyut 7 & $*$ & $*$ & $*$ & & $*$ & $*$ & \\
\hline TerNov & $*$ & * & & $*$ & & * & \\
\hline Apollo 11 & $*$ & $*$ & * & & & $*$ & \\
\hline DomEx & & $*$ & & * & & $*$ & \\
\hline Frozen & & & * & & * & & \\
\hline IGY & & $*$ & & & & & \\
\hline Steger & & & & & & & \\
\hline
\end{tabular}

\section{ACKNOWLEDGMENTS}

This research was supported in part by National Science Foundation grants SBR-9729957 and SES-9944042.

\section{CONTACT}

Direct all correspondence to Dr. Marilyn Dudley-Rowley, c/o OPS-Alaska, 1030 Carl Shealy Road, Irmo, South Carolina 29063; 803/732-3604; Leona@Martiana.org; www.Martiana.org.

\section{REFERENCES}

Aldrin, E. 1973. Return to Earth, Random House, New York.

Back, J.D. ed. 1992. The Quiet Land: The Diaries of Frank Debenham, Member of the British Antarctic Expedition (1910-1913), Erskine Press, Norfolk.

Baddeley, A.D. 1972. "Selective Attention and Performance in Dangerous Environments." The British Journal of Psychology 63(4), 537-546.

Bishop, S. 2000. Unpublished remarks made in the "Performance/Human Factors - 3" Session at the 13th Humans in Space Symposium, Santorini, Greece, May 25.

Blau, P. 1977. Inequality and Heterogeneity: A Primitive Theory of Social Structure, The Free Press, New York.

Burrough, B. 1998. Dragonfly: NASA and the Crisis Aboard Mir, HarperCollins Publishers, Inc., New York.

CBC News: E-mail News Digest. "Canadian Fends Off Advances in Space Module." WebPosted Wed., March 22, 2000.

Csikszentmihalyi, M. 1991. Flow: The Psychology of

Optimal Experience, HarperCollins, New York.

Dudley-Rowley, M. 1997. "Deviance Among Expeditioners: Defining the Off-Nominal Act Among Space and Polar Field Analogs." J. Human Performance in Extreme Environments 2(1), 119-127.

Dudley-Rowley, M. 2000 The Effects of Size and Heterogeneity of Crew and Mission Duration on the Deviant Behavior and Performance of Team Personnel In Space and Analog Polar Environments: a Pilot Study, UMI, Ann Arbor.

Dudley-Rowley, M., Patrick Nolan, Sheryl Bishop, Kristin Farry, and Thomas Gangale. 2001. "Ten Missions, Two Studies: Crew Composition, Time, and Subjective Experience in Mars-Analog Expeditions." Proceedings of the Third International Convention of the Mars Society, Univelt, Incorporated, San Diego.

Endsley, M.R. and C.A. Bolstad. 1994. "Individual Differences in Pilot Situation Awareness." International Journal of Aviation Psychology 4, 241-264.

Evans, Gary W., Daniel Stokols, and Sybil Carrere. 1988. Human Adaptation to Isolated and Confined Environments: Preliminary Findings of a Seven Month Antarctic Winter-over Human Factors Study, NASA, Contract NAG2-387, NASA Contractor Report 177499.

Greely, A. 1886. Three Years of Arctic Service: An Account of the Lady Franklin Bay Expedition of 18811884, and the Attainment of the Farthest North, Scribner, New York.

Guly, C. 2000. "Terrified Researcher 'Slept With a Knife': Russian Commander's Forceful Advances Turn Space Isolation Study Into Nightmare." Ottawa Citizen, March 24.

Lebedev, Valentin. 1988. Diary of a Cosmonaut: 211 Days in Space, Phytoresource Research, Inc., Texas.

Lewis, D. and M. George. 1987. Icebound in Antarctica. W.W. Norton \& Company, New York. 
Lovell, J. and Jeffrey Kluger. 1994. Apollo 13 [Lost Moon: The Perilous Voyage of Apollo 13], Pocket Books, New York.

Mayhew, B.H. and R. Levinger. 1976. "Size and the Density of Interaction Among Social Aggregates." American Journal of Sociology 81, 1017-1049.

Pearce, R. 1930. Marooned in the Arctic: Diary of the Dominion Explorers' Expedition to the Arctic, August to December 1929, The Northern Miner, Winnipeg.

Pierce, C. M. 1991. "Theoretical Approaches to Adaptation to Antarctica and Space" in From Antarctica to Outer Space: Life in Isolation and Confinement, A.A. Harrison, Y.A. Clearwater, and C.P. McKay (Eds.). Springer-Verlag, New York, 125-134.

Pirrit, J. 1967. Across West Antarctica, John Smith \& Son, Glasgow.

Sandal, G.M. 2000. "Dynamics of Interpersonal Tension on a Simulated International Space Station." Presented in the "Performance/Human Factors - 3" Session at the 13th Humans in Space Symposium, Santorini, Greece, May 25.

Stefansson, V. 1925. The Adventure of Wrangel Island, the MacMillan Company, New

Steger, W. and J. Bowermaster. 1992. Crossing Antarctica, Alfred A. Knopf, New York .

Stuster, Jack. 1996. Bold Endeavors, Naval Institute Press, Annapolis.

Zubrin, Robert and Richard Wagner. 1996. The Case for Mars: the Plan to Settle the Red Planet and Why We

Must, The Free Press, New York. 